\title{
On the Prudence of Rewarding A While Hoping for $B$
}

\author{
Wendelin Schnedler
}

April 2003

\begin{abstract}
In multiple-task hidden-action models, the (mis-)allocation of effort may play an important role for benefit creation. Signals which capture this benefit and which are used in incentive schemes should thus not only be judged by the noise and the associated costs but also by the mis -allocation which they induce. How can mis-allocation be measured? This article presents two requirements for such a measure (invariance and monotonicity) and analyses whether they are met by proposed measures of mis-allocation. All examined measures assert that an unbiased signal for benefit leads to the lowest mis-allocation. Here, the signal leading to the lowest mis-allocation is computed and shown to be unbiased for the benefit only under restrictive assumptions on the cost function (equal marginal costs for different tasks). Generally, using an unbiased signal does not imply the lowest mis -allocation.
\end{abstract}

Keywords: multitasking, LEN-model, hidden-action, moral hazard

JEL-Codes: M52, D82, M41.

\section{Acknowledgements}

We thank the Leverhulme Trust for funding this research.

\author{
Address for Correspondence \\ Department of Economics \\ University of Bristol \\ 12 Priory Road \\ Bristol \\ BS8 1TN \\ W.Schnedler@bristol.ac.uk
}




\section{Introduction}

Incentive schemes sometimes lead to outcomes which are not intended. The reason is often that the incentive scheme induces the worker to concentrate on activities which are honoured by the incentive scheme while neglecting important other activities. Loosely speaking, the benefit $B$ received by the employer and the signal A, which is used to reward the worker, are not aligned. In other words, the mis-allocation of effort may be traced back to the "Folly of rewarding A, while hoping for B" (Kerr 1975).

However, there might be good reasons why incentives and benefit are not aligned. Delegating an activity and using a non-aligned incentive scheme can work as a commitment device - for example to stick to an aggressive behaviour in an oligopolistic competition (Sklivas 1987, Fershtman and Judd 1987). Also, misalignment may be deliberately embraced when it is tied to a reduction in uncertainty (Datar, Kulp, and Lambert 2001, Baker 2000 and 2002, Schnedler 2002) . The present article is concerned with neither of these explanations. Rather it asks the more basic questions: Is a signal, which is aligned with the benefit, desirable for an incentive scheme in a multiple-task context? In particular: Does it lead to low mis-allocation of effort?

The problem of mis-allocation only arises because effort cannot be contractually fixed. The natural framework to study such situations is the hidden-action model with its well known trade-off between uncertainty and incentives (Holmström 1979). ${ }^{1}$ If the hidden-action model is extended to multiple actions (tasks), distortions of effort allocation may occur and have devastating effects as firstly shown by Holmström and Milgrom (1991) and supported by several real-life cases (see e.g. Prendergast 1999 or Baker 2002). In multiple-task hidden action models, the trade-off does not only involve uncertainty and the level of effort but also the allocation of effort. This complicates the ranking of signals according to signal properties: While in single-task hidden-action models the signal with a lower uncertainty is clearly preferable, in multiple-task hidden-action models signals can in principle also be ordered by the mis-allocation which they induce. Attempts to measure mis-allocation are either not simple (Schnedler 2002) or they apply only to a specific framework (Feltham and Xie 1994 and Baker 2002). Moreover, measures are often based on ad-hoc considerations and hence it is not clear whether

\footnotetext{
${ }^{1}$ For a recent critical assessment of this trade-off see Prendergast (2000).
} 
they actually quantify "mis-allocation".

Here, we focus on the framework for which most of the measures are devised, the multiple-task linear-exponential-normal model (LEN model), and define two simple properties which a measure of mis-allocation should have. First, keeping the effect of uncertainty fixed, two signals which lead to the same surplus should be associated with the same mis-allocation (invariance). Second, a larger mis-allocation should be associated with a lower surplus when the effect of uncertainty is held constant (monotonicity). We check whether the measures of Feltham and Xie (1994) and Baker (2002) exhibit these properties and modify the measure of Baker.

All examined measures confirm the basic intuition that "rewarding for A, while hoping for B" is foolish: They assert that an unbiased signal for the benefit B leads to the lowest mis-allocation of effort. A special unbiased signal is the congruent signal. For a congruent signal, the marginal effect of effort on the expected signal is identical to the marginal productivity of effort. Hence, it seems as if congruent signals set the right incentives for effort allocation.

To test this intuition, we compute the signal leading to the lowest mis-allocation. Surprisingly, it turns out that this signal is neither necessarily congruent nor unbiased. In other words, a signal which puts more emphasis on some efforts than a congruent signal may yield a higher surplus - even though both signals impose the same uncertainty on the agent. Despite hoping for the benefit $\mathrm{B}$, it thus pays rewarding according to a biased signal A rather than a signal which unbiasedly reflects $B$.

The reason for this result is the following. As soon as uncertainty and risk aversion are present, the worker will be insured and thus not fully internalise the benefits of effort. While this has a clear and known effect on the level of effort, it also affects the allocation of effort between tasks. To counteract this effect, signals which put more emphasis on certain efforts are more desirable than unbiased or congruent signals.

The remainder of the paper is structured as follows: Section 2 presents a generalised version of the multiple task LEN-model. Section 3 provides the solution to the incentive programme. Then, desirable properties of measures for mis-allocation (invariance, monotonicity) are defined in section 4. Section 5 re- 
views the mis-allocation measures proposed by Feltham and Xie (1994) and Baker (2002) and checks whether they exhibit the desired properties. In section 6 , the signal leading to the lowest mis-allocation is determined and a sufficient condition is given when this signal is unbiased. Section 7 presents an example where biased signals are optimal. Finally, section 8 concludes.

\section{The model}

The model features two economic decision makers: a principal and an agent. For simplicity the principal will be referred to by female pronouns while the agent will be referred to by male pronouns. The agent chooses a $n$-dimensional effort vector $e$. While the costs of the effort $C(e)$ are incurred by the agent, the benefit $B(e)$ accrues to the principal. Presumably, there is some pareto-optimal effort choice $e^{*}$ which maximises $B(e)-C(e)$. If effort is verifiable at court, principal and agent will agree on a contract in which the agent delivers $e^{*}$ and the principal remunerates him. If, however, effort is not verifiable, such a contract cannot be enforced by court; principal and agent must rely on other means to internalise the externality which the agent imposes on the principal. For example, there may be a signal $S(e)$ available, which conveys information about effort $e$ because its distribution depends on $e$. By conditioning transfers on the observed signal, the principal can reward and punish particular effort choices. Signals are often imperfect substitutes for a contract, which directly stipulates effort, because they may impose uncertainty on a risk-averse agent and because they do not necessarily reflect the different importance of the various dimensions of effort.

The particular model which will be employed here is the linear-exponential-normal model or LEN-model, which is typically used when closed form solutions are needed or when dealing with multiple tasks (e.g. Holmström and Milgrom 1991, Feltham and Xie 1994, Baker 2002) The name of the model, which was coined by Spremann (1987), reflects the important assumptions characterising this model. First, transfers to the agent $T(S(e))$ are assumed to be linear functions of the signal: $T(S(e))=w_{0}+w_{1} S(e)$, where the term $w_{0}$ represents the base wage paid to the agent which is independent of the service and $w_{1}$ is the performance wage rate. If $w_{1}$ is large in absolute terms this indicates a high degree of "incentivisation". It should be outlined that linear transfers are generally not optimal (Mirrlees 1975, see also Wagenhofer and Ewert 1993 and the criticism of their work by Breuer 1993). However, besides leading to a closed-form solution, linear transfers 
are sometimes justified on the basis of their robustness in complex environments (Holmström and Milgrom 1987). Second, the utility of the agent is exponential: $U(v)=-e^{(-v r)}$, where $v$ is the difference between the transfers received and the costs incurred by the agent $(v=T(S(e))-C(e))$ and $r$ is the Arrow-Pratt measure for risk aversion. The larger this measure the more risk-averse is the agent. Finally, the noise term in signals is assumed to be normally distributed.

Based the LEN-model Feltham and Xie (1994) devise a hidden-action model with multiple efforts. In their model, benefit is a linear function of efforts:

$$
B(e)=\sum_{i=1}^{n} \beta_{i} e_{i}=\left(\beta_{1}, \ldots, \beta_{n}\right)\left(\begin{array}{c}
e_{1} \\
\vdots \\
e_{n}
\end{array}\right)=\beta^{\prime} e
$$

where the prime indicates the transpose of a vector. The components of the benefit coefficient vector $\beta$ represent the marginal productivity of the respective effort dimension. Similarly, signals are defined as linear functions of effort:

$$
S(e)=\sum_{i=1}^{n} b_{i} e_{i}+\varepsilon=\left(b_{1}, \ldots, b_{n}\right)\left(\begin{array}{c}
e_{1} \\
\vdots \\
e_{n}
\end{array}\right)+\varepsilon=b^{\prime} e+\varepsilon
$$

where $\varepsilon$ is a normally distributed error term with mean zero and variance $\sigma^{2}$. Note, that a signal is completely characterised by its coefficient vector and variance: $\left(b, \sigma^{2}\right)$. The components of the signal coefficient vector indicate the marginal effect of the respective effort dimension on the expected signal. The similar structure of the benefit and the signal is attractive because we can hope to trace misallocation back to differences in the two coefficient vectors $b$ and $\beta$. Indeed, the measures of mis-allocation which are proposed by by Feltham and Xie (1994) as well as Baker (2002) are based on a comparison of these two vectors.

In contrast to Feltham and Xie (1994) and Baker (2002), a more general cost function for effort is assumed here:

$$
C(e)=\sum_{i=1}^{n} \sum_{j=1}^{n} e_{i} c_{i j} e_{j}=\left(e_{1}, \ldots, e_{n}\right)\left(\begin{array}{ccc}
c_{11} & \cdots & c_{1 n} \\
\vdots & \ddots & \vdots \\
c_{n 1} & \cdots & c_{n n}
\end{array}\right)\left(\begin{array}{c}
e_{1} \\
\vdots \\
e_{n}
\end{array}\right)=e^{\prime} C e
$$

The matrix $C$ is assumed to be symmetric and non-negative definite, so that costs are always positive: $e^{\prime} C e>0$ if $e \neq(0, \ldots, 0)^{\prime}$. The cost function of the previously 
mentioned articles can be obtained by setting $C=\frac{1}{2} I$, where $I$ is the identity matrix. Later, it will turn out that some results which are true with the simpler cost function do not hold in the more general case.

\section{Incentive problem and solution}

What is the maximal surplus that principal and agent can obtain when they cannot contract on effort in the model above? For the answer, we follow the standard procedure (see e.g. Macho-Stadler and Pérez-Castrillo 1997) of assigning the role of the mechanism designer to the principal and let her receive any benefit from the operation of the incentive scheme. A side condition (participation constraint) ensures that the benefit is indeed generated by the mechanism and not by exploiting the agent. Finally, the rationality of the agent with respect to the effort is represented by a second side condition (incentive constraint). Overall, the maximisation programme takes the form:

$$
\begin{array}{cl} 
& \max w_{0}, w_{1} B(e)-w_{0}-w_{1} S(e) \\
\text { such that } & e \in \operatorname{argmax}_{\tilde{e}} \mathrm{E}\left(U\left(w_{0}+w_{1} S(\tilde{e})-C(\tilde{e})\right)\right) \\
\text { and } & \mathrm{E}\left(U\left(w_{0}+w_{1} S(e)-C(e)\right)\right) \geq 0 .
\end{array}
$$

Using the linearity of the signal, the normality distribution of the noise, and the shape of the utility function, the base wage can be chosen to compensate the agent for any harmful effects of uncertainty $\left(w_{0}=C(e)-w_{1} b^{\prime} e+w_{1}^{2} r \sigma^{2}\right)$, so that the participation constraint can be eliminated from the programme (see e.g. Salanié 1998 or Kräkel 1999). Recalling the definitions of signal, benefit, and costs, the programme then simplifies to:

$$
\begin{gathered}
\max w_{1} \beta^{\prime} e-e^{\prime} C e-\frac{w_{1}^{2}}{2} r \sigma^{2} \\
\text { such that } e \in \operatorname{argmax}_{\tilde{e}} \mathrm{E}\left(U\left(w_{0}+w_{1} b^{\prime} \tilde{e}-\tilde{e}^{\prime} C \tilde{e}\right)\right) .
\end{gathered}
$$

To find the solution to this programme, we first consider the effort choice problem of the agent for a given incentive scheme $\left(w_{0}, w_{1}\right)$. The computed efforts are then replaced in the main objective function to determine the choice of $w_{1}$. Because the utility function is monotonous in the received wage minus the effort costs, the agent chooses effort so as to maximise this difference:

$$
\max _{e} w_{1} b^{\prime} e-e^{\prime} C e
$$


The objective function is concave because the second derivative is a symmetrical, negative definite matrix $(-C)$. Thus, the maximiser can be determined by the first-order condition. Solving for $e$ yields:

$$
e=\frac{w_{1}}{2} C^{-1} b
$$

Note, that $C^{-1}$ exists because $C$ is positive definite. Recalling that $B(e)=\beta^{\prime} e$ the maximisation programme becomes:

$$
\max _{w_{1}} \beta^{\prime} e-e^{\prime} C e-\frac{w_{1}^{2}}{2} r \sigma^{2} .
$$

Replacing $e$ by the optimal effort yields the following expression for the objective function which now only depends on the optimal performance wage rate:

$$
\max _{w_{1}} \frac{w_{1}}{2} \beta^{\prime} C^{-1} b-\frac{w_{1}^{2}}{2^{2}} b^{\prime} C^{-1} b-\frac{w_{1}^{2}}{2} r \sigma^{2} .
$$

Again, the objective function is concave and solving the first order condition gives the maximiser:

$$
w_{1}=\frac{\beta^{\prime} C^{-1} b}{b^{\prime} C^{-1} b+2 r \sigma^{2}}
$$

Using the optimal wage rate in (7) finally results in an expression for the surplus which can be generated from an incentive scheme based on the signal $\left(b, \sigma^{2}\right)$ :

$$
\phi\left(b, \sigma^{2}\right)=\frac{1}{4} \frac{b^{\prime} C^{-1} \beta \beta^{\prime} C^{-1} b}{b^{\prime} C^{-1} b+2 \sigma^{2} r} .
$$

Since the surplus depends on the signal parameters, it is reasonable to ask the following question: What is a good signal? What parameter values characterise such a signal? In single-task models, where the only problem is the noise of the signal, the variance is the crucial parameter: A lower variance implies less compensation for uncertainty and is therefore attractive. Uncertainty is still important in a multiple-task model. Now, there is also the problem of effort allocation and some signals may be more useful to achieve a desired allocation. A particularly promising candidate is a signal which unbiasedly reflects the benefit that the principal draws from the effort.

In statistics and econometrics, an estimator is called unbiased for an unknown parameter, if its expected value coincides with the parameter. The following definition is the respective equivalent in a hidden action model with linear contracts. 
Definition 1 (Unbiased benefit signal). A signal is called unbiased for the benefit if its expected value equals a multiple of the benefit independently from the exerted effort:

$$
E(S(e))=b^{\prime} e=k \beta^{\prime} e=k B(e) \text { for some fixed } k \text { and all } e .
$$

In contrast to the econometrical and statistical definition, we allow the expected value to be a multiple of the benefit. We do so because the signal will be used in a linear wage scheme, so that the factor $k$ can be offset by choosing a piece-wage rate which is re-standardised: $\tilde{w}_{1}=\frac{w_{1}}{k}$.

A second way of characterising a signal is by the relationship between signal and benefit coefficient vector.

Definition 2 (Congruent signal). A signal is congruent if $b=\beta$.

Are congruence and unbiasedness related?

Result 1. A signal $\left(b, \sigma^{2}\right)$ is unbiased for the benefit if and only if the signal coefficient vector is a multiple of the marginal benefit vector $(b=k \beta)$ for some $k$.

Proof. If $b^{\prime} e=k \beta^{\prime} e$ for all $e$, then it is also valid for the unity vector $e^{i}$ which has a one at the $i$-th component $e^{i}=(0, \ldots, 0,1,0, \ldots, 0)$. It follows that $b_{i}=k \beta_{i}$. Conversely, $b=k \beta$ directly implies that $b^{\prime} e=k \beta^{\prime} e$.

By choosing $k=1$, this result uncovers the relation between congruence and unbiasedness: Any congruent signal is unbiased.

An unbiased signal - in particular a congruent signal - seems to be a good candidate to replace the non-contractible objective in an optimal incentive scheme because it correctly reflects the relative weight of different efforts in the benefit. Later, we will derive a condition under which this intuition is valid. But before we can do so, we have to characterise when signals are "good" and incentive schemes are "optimal" with respect to mis-allocation. There are several ways to assess the mis-allocation of a signal. The next section provides two criteria to distinguish between those ways.

\section{Properties of mis-allocation measures}

If we desire to rank signals according to the induced mis-allocation, we have to ensure that uncertainty does not interfere. This cannot be achieved by simply fixing the variance; it is the relation between the informative and the non-informative 
part of the signal which matters. If, for example, the variance is kept constant and the length of the signal coefficient vector is increased, the informative content also increases and the effect of uncertainty on the surplus decreases - see equation (9). Apparently, the informative content must be related to the variance in order to effectively control for uncertainty. This suggests the following definition.

Definition 3 (Signal-noise ratio). The squared length of the signal coefficient vector divided by the variance is called signal-noise ratio: $\rho:=\frac{b^{\prime} b}{\sigma^{2}}$.

Is this a sensible definition? Can we eliminate the effect of uncertainty by fixing the signal-noise ratio?

Result 2. For all signals with signal-noise ratio $\rho$, the effect of uncertainty on the surplus is constant.

Proof. Take the surplus generated and replace $\sigma^{2}$ by $\frac{b^{\prime} b}{\rho}$, this yields the surplus:

$$
\phi\left(b, \frac{\rho}{b^{\prime} b}\right)=\frac{1}{4} \frac{b^{\prime} C^{-1} \beta \beta^{\prime} C^{-1} b}{b^{\prime}\left(C^{-1}+2 \frac{r}{\rho} I\right) b},
$$

which is constant in $\sigma^{2}$ and does not change with the length of the vector $b ; b$ can be standardised to a fixed length and the standardisation factors in the numerator cancel with those in the denominator.

Hence, we can fix the effect of uncertainty using the notion of a constant signalnoise ratio. This will later enable us to identify and control for the effect of misallocation.

Mis-allocation measures should only pick up the effect of signals on the allocation of effort. Consider two signals with the same signal-noise ratio. Hence, all differences between these signals result from a different allocation of effort. If these signals lead to the same surplus, there is no reason why the mis-allocation should be different. In fact, if a mis-allocation measure would assign different values to two such signals, it ranks them without a proper foundation. This idea is summarised in the following definitions.

Definition 4 (Invariance and Scale-Invariance). Consider two signals $\left(b, \sigma^{2}\right)$ and $\left(\tilde{b}, \tilde{\sigma}^{2}\right)$. A measure for mis-allocation $\delta(\cdot, \cdot)$ is called invariant if and only if

$$
\rho\left(b, \sigma^{2}\right)=\rho\left(\tilde{b}, \tilde{\sigma}^{2}\right) \text { and } \phi\left(b, \sigma^{2}\right)=\phi\left(\tilde{b}, \tilde{\sigma}^{2}\right) \text { implies } \delta\left(b, \sigma^{2}\right)=\delta\left(\tilde{b}, \tilde{\sigma}^{2}\right) \text {. }
$$


A measure for mis-allocation $\delta(\cdot, \cdot)$ is called scale-invariant if and only if

$$
\delta\left(b, \sigma^{2}\right)=\delta\left(q b, q^{2} \sigma^{2}\right)
$$

Scale-invariance captures the idea that a mere change of the scale on which a signal is measured should not have an effect on the mis-allocation of effort. Because re-scaling neither alters the effect of uncertainty $\rho$ nor changes the surplus which can be obtained $\phi$, an invariant mis-allocation measure $\delta$ must assign the same value to the rescaled signal and is hence scale-invariant:

Result 3. Any invariant mis-allocation measure is also scale-invariant.

The invariance property alone is not very helpful to identify a good mis-allocation measure. By this criterion, any constant function qualifies as "good". However, a meaningful mis-allocation measure should vary with the properties of signals. A simple requirement, which adds meaning to a mis-allocation measure, can be defined by relating the measure to surplus. In particular, when comparing two signals with the same signal-noise-ratio (so that the effect of uncertainty is held constant), the signal leading to a lower mis-allocation should generate the higher surplus. This idea is expressed in the following definition.

Definition 5 (Monotonicity). A mis-allocation measure $\delta(\cdot, \cdot)$ is called monotonous if it ranks any two signals $\left(b, \sigma^{2}\right)$ and $\left(\tilde{b}, \tilde{\sigma}^{2}\right)$ with the same signal-noise ratio $\rho=\tilde{\rho}$ according to the surplus $\phi(\cdot, \cdot)$ which can be generated with these signals:

$$
\delta\left(b, \sigma^{2}\right)>\delta\left(\tilde{b}, \tilde{\sigma}^{2}\right) \Leftrightarrow \phi\left(b, \sigma^{2}\right)<\phi\left(\tilde{b}, \tilde{\sigma}^{2}\right) \text { given } \rho=\tilde{\rho} .
$$

If faced with a decision between two signals with the same signal-noise ratio, the preferable signal must be the one with a lower mis-allocation. Of course, this definition is based on the idea that the second-best surplus is only affected by two things: the effect of uncertainty and the effect of mis-allocation. If there were a third effect than eliminating the effect of uncertainty would not necessarily imply that the effect of mis-allocation must explain differences in surplus.

The two elementary properties of mis-allocation measures introduced in this section, invariance and monotonicity, already have far reaching implications and will prove helpful later to construct and discard mis-allocation measures. 


\section{Mis-allocation Measures}

In this section, we present two measures for effort mis-allocation, check whether they exhibit the properties of invariance and monotonicity, and propose a third measure.

\subsection{Feltham's and Xie's measure}

Using the same model as in this paper with equally costly tasks $\left(C=\frac{1}{2} I\right)$, Feltham and Xie (1994) observe that the relative effort expended in any two tasks is equivalent to the marginal effects of these efforts on the expected signal:

$$
\frac{e_{i}}{e_{j}}=\frac{b_{i}}{b_{j}},
$$

which can be seen from equation (6) when $C$ is replaced accordingly. At the same time and under the same assumption about $C$, the pareto-optimal effort is equal to the marginal productivity $\left(e_{i}^{*}=b_{i}\right)$, so that the ratio of pareto-optimal efforts is equal to the ratio of marginal productivities:

$$
\frac{e_{i}^{*}}{e_{j}^{*}}=\frac{\beta_{i}}{\beta_{j}} .
$$

Now, Feltham and Xie (1994) regard "mis-allocation" to result from a deviation of the induced effort ratio from the first-best effort ratio. Then, signals which assign the same relative weight to efforts as in the benefit function $\left(\frac{b_{i}}{b_{j}}=\frac{\beta_{i}}{\beta_{j}}\right)$ are attractive because they lead to an induced effort ratio which is identical to the first-best effort ratio:

$$
\frac{e_{i}}{e_{j}}=\frac{e_{i}^{*}}{e_{j}^{*}} .
$$

Consequently, a mis-allocation measure can be devised by comparing the signal coefficients $b$ with the benefit coefficients $\beta$. For a model with two efforts, Feltham and Xie (1994) propose the following definition.

Definition 6 (Feltham and Xie's mis-allocation measure). If $\left(b, \sigma^{2}\right)$ is a signal, its mis-allocation according to Feltham and Xie is:

$$
\delta^{F X}\left(b, \sigma^{2}\right):=\left(\beta_{1} b_{2}-\beta_{2} b_{1}\right)^{2} .
$$

What is the signal with the lowest mis-allocation according to this measure? 
Result 4. The lowest mis-allocation according to $\delta^{F X}$ is achieved by an unbiased signal.

Proof. If $b$ is unbiased, it holds that $b=k \beta$. Replacing the components $b_{1}$ by $k \beta_{1}$ and $b_{2}$ by $k \beta_{2}$, we obtain the value $\delta^{\mathrm{FX}}\left(k \beta, \sigma^{2}\right)=0$ because the measure is always positive, this is the lowest value which can be reached.

Thus, the measure of Feltham and Xie confirms the intuition that unbiased and congruent signals are preferable due to their allocation properties. However, the measure is problematic:

Result 5. The mis-allocation measure $\delta^{F X}$ is neither invariant and nor monotonous.

Proof. Consider the signal $\left(b, \sigma^{2}\right)$ and the re-scaled signal $\left(q b, q^{2} \sigma^{2}\right)$ with $q>1$. The respective mis-allocation is:

$$
\delta^{\mathrm{FX}}\left(b, \sigma^{2}\right)=\left(\beta_{1} b_{2}-\beta_{2} b_{1}\right)^{2}<\left(\beta_{1} b_{2}-\beta_{2} b_{1}\right)^{2} q^{2}=\delta^{\mathrm{FX}}\left(q b, q^{2} \sigma^{2}\right) .
$$

Thus, the re-scaled signal is not assigned the same value as the original signal and the measure is not scale-invariant. As scale-invariance is a special case of invariance, the measure is not invariant either. Also, the measure detects a difference in mis-allocation where there is no respective change in surplus: $\delta^{\mathrm{FX}}\left(b, \sigma^{2}\right)<$ $\delta^{\mathrm{FX}}\left(q b, q^{2} \sigma^{2}\right)$ but $\phi\left(b, \sigma^{2}\right)=\phi\left(q b, q^{2} \sigma^{2}\right)$ while $\rho\left(b, \sigma^{2}\right)=\rho\left(q b, q^{2} \sigma^{2}\right)$. So, the measure is not monotonous.

While the measure $\delta^{\mathrm{FX}}$ might convey some intuition about effort mis-allocation, it does not feature the two simple properties of monotonicity and invariance.

\subsection{Baker's measure}

Baker (2002) comes forward with a different mis-allocation measure, which is based on an examination of the optimal performance wage rate $w_{1}$, which we calculated in equation (8), where again identical costs for efforts are assumed $\left(C=\frac{1}{2} I\right):^{2}$

$$
w_{1}=\frac{\beta^{\prime} b}{b^{\prime} b+r \sigma^{2}}=\frac{\sqrt{b^{\prime} b} \sqrt{\beta^{\prime} \beta} \cos (\gamma)}{b^{\prime} b+r \sigma^{2}},
$$

where $\gamma$ is the angle between the benefit and signal coefficient vector and where the equality follows from $\cos (\gamma)=\frac{b^{\prime} \beta}{\sqrt{b^{\prime} b} \sqrt{\beta^{\prime} \beta}}$. Thus, the intensity to which the

\footnotetext{
${ }^{2}$ For the original formula in Baker, one needs to assume that the length of the two vectors is identical $F:=\sqrt{b^{\prime} b}=\sqrt{\beta^{\prime} \beta}$.
} 
signal is used depends on the cosine of the angle between the vectors and Baker (2002) suggests to use this term as a mis-allocation measure.

Definition 7 (Baker's mis-allocation measure). If $\gamma$ is the angle between the signal and benefit coefficient vector, the mis-allocation according to Baker is:

$$
\delta^{B}:=\cos (\gamma)
$$

Differently from Feltham and Xie's measure, Baker's measure decreases in the mis-allocation and is hence a measure of good rather than bad allocation. This mere semantic difference aside, the measure shares an important feature with Feltham and Xie's measure:

Result 6. The lowest mis-allocation according to $\delta^{B}$ is achieved by an unbiased signal.

Proof. Given $b=k \beta$, the angle between $b$ and $\beta$ is zero and the cosine attains its maximal value of one - indicating the lowest mis-allocation.

Thus, Baker's measure supports the initially mentioned intuition and favours unbiased and congruent signals over biased signals.

The measure of Baker can be also motivated by appealing to the idea of scaleinvariance. Consider the signal class with constant signal-noise ratio $\rho$. Any signal from this class can be represented by a signal with a signal coefficient vector $b$, the length of which is equal to the marginal productivity vector $\beta$. This can simply be achieved by multiplying the signal by $q=\sqrt{\frac{\beta^{\prime} \beta}{b^{\prime} b}}$. By the scale-invariance property, this change should have no effect on the measured mis-allocation.

If we depict the benefit and signal coefficient vector in a two-dimensional plane (see figure 1), a change in scale implies a change in the length of the signal coefficient vector. According to the scale-invariance property, this change should have no effect on the mis-allocation. Indeed, the angle between the two vectors remains constant and contains information about the relationship between signal and benefit. This suggests that the angle can be used to construct a measure for mis-allocation.

Because signal coefficient vectors which point into the same direction create the same surplus, the invariance property requires that these vectors get assigned the 


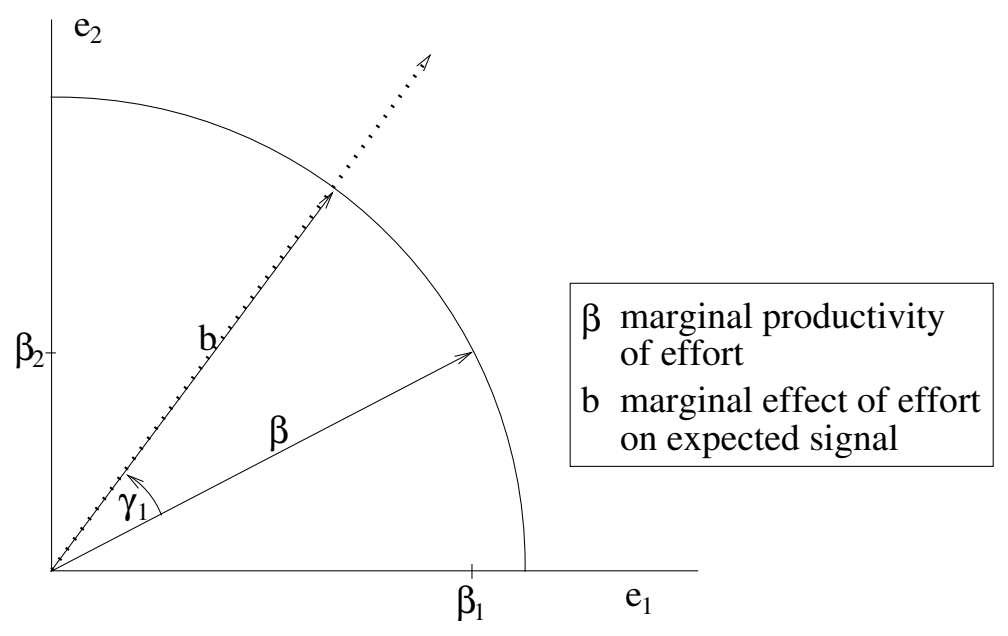

Figure 1: Effect of Scale-changes

Changing the scale of the signal does not influence the angle between signal and benefit coefficient vector.

same value for mis-allocation. For an angle-based measure, this implies that values should repeat themselves every $2 \pi$ or every $360^{\circ}$. Trigonometric functions have this period. This explains why to consider the cosine of the angle between the signal and benefit coefficient vector. Hence, Baker's measure naturally arises from invariance considerations. Does this imply that the measure is invariant?

Result 7. $\delta^{B}$ is neither invariant nor monotonous.

Proof. Consider the signals $\left(b, \sigma^{2}\right)$ and $\left(-b, \sigma^{2}\right)$ which have the same signal-noise ratio and lead to the same surplus. Let $\gamma \in(-\pi, \pi)$ be the angle between $b$ and $\beta$. Then, the angle between $-b$ and $\beta$ is $\pi+\gamma$. The respective mis-allocation measure is: $\delta^{\mathrm{B}}\left(b, \sigma^{2}\right)=\cos (\gamma)>-\cos (\gamma)=\cos (\pi+\gamma)=\delta^{\mathrm{B}}\left(-b, \sigma^{2}\right)$. This has two implications: First, a change in the measure occurs as a result of re-scaling and the measure is not invariant. Second, because a change in the value of misallocation is not reflected in surplus the measure is not monotonous.

\subsection{A modification of Baker's measure}

The counter-example which destroys invariance and monotonicity of the cosine as a mis-allocation measure is based on two signals pointing in opposing directions. 


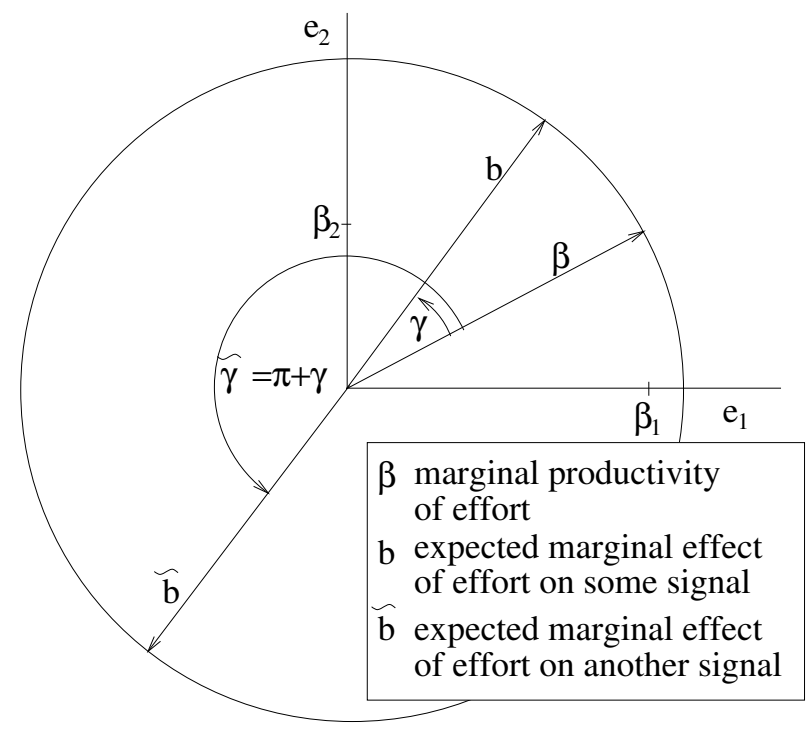

Figure 2: Periodicity of mis-allocation

Two signal vectors which point in the same direction $(2 \pi$ apart) or in opposite directions ( $\pi$ apart) generate the same surplus and should be assigned the same mis-allocation.

The respective coefficient vectors generate the same surplus because the signal can be multiplied with minus one before using it. Such opposing vectors are $\pi$ apart and generate the same surplus, thus an angle-based mis-allocation measure should have period $\pi$ rather than $2 \pi$. The absolute value of the cosine has this property. This hints to modify Baker's by using the absolute value:

$$
\delta^{\mathrm{M}}:=|\cos (\gamma)| \text {. }
$$

A second motivation for the modified measure is similar to Baker's original idea about "incentive intensity". Incentive intensity is not only given when the wage rate $w_{1}$ is positive and large, it is also present when the wage rate is negative and large. Then, the agent is motivated to work hard in order to avoid punishment. So the intensity of incentives should be measured in terms of the absolute value of the wage $\left|w_{1}\right|$ rather than the actual value $w_{1}$. Doing so, and following Baker's argument, the absolute value of the cosine replaces the cosine as a measure of mis-allocation. The so-obtained measure features several defined properties:

Result 8. The mis-allocation measure $\delta^{M}$ is scale-invariant. Given that costs for tasks are equal $(C=c I)$, it is also invariant and monotonous. 
Proof. Let $\tilde{b}=q b$ be a rescaling of $b$ and $\gamma$ be the angle between $b$ and $\beta$. Then, the angle between $\tilde{b}$ and $\beta$ is $\tilde{\gamma}=\pi k+\gamma$ for some natural number $k$. The respective distortion measures are:

$$
\delta^{\mathrm{M}}\left(b, \sigma^{2}\right)=|\cos (\gamma)|=|\cos (\gamma+\pi k)|=\delta^{\mathrm{M}}\left(q b, q^{2} \sigma^{2}\right),
$$

where the central equality results from the fact that the absolute value of the cosine has period $\pi$. Consequently, the measure is scale-invariant. Given equal costs $(C=c I)$, the surplus generated by a signal $\left(b, \frac{\rho}{b^{\prime} b}\right)$ is:

$$
\phi\left(b, \frac{\rho}{b^{\prime} b}\right)=\frac{1}{4} \frac{c^{-2} b^{\prime} \beta \beta^{\prime} b}{\left(c^{-1}+2 \frac{r}{\rho}\right) b^{\prime} b}=\frac{b^{\prime} \beta \beta^{\prime} b}{4 c^{2}\left(c^{-1}+2 \frac{r}{\rho}\right) b^{\prime} b} \frac{\beta^{\prime} \beta}{\beta^{\prime} \beta}=\frac{\cos (\gamma)^{2} \beta^{\prime} \beta}{4 c^{2}\left(c^{-1}+2 \frac{r}{\rho}\right)} .
$$

Note, that the signal enters this quantity only via $\gamma$. Moreover, the surplus is strictly increasing in $\delta^{\mathrm{M}}=|\cos \gamma|$ and attains it maximum for $\gamma=k \pi, k=0,1, \ldots$ Thus, the surplus varies if and only if $\delta^{\mathrm{M}}$ varies: The measure is invariant and monotonous.

The modified measure retains the property of Baker's measure that unbiased and congruent signals yield the lowest mis-allocation: The angle between an unbiased signal and benefit vector is either zero or a multiple of $\pi$ and the absolute value of the cosine for this values yields the maximum.

All considered mis-allocation measures assess the mis-allocation to be lowest when using unbiased signals. So, one is tempted to conclude that unbiased signals are indeed preferable from a mis-allocation standpoint. Of course, this statement hinges on the monotonicity of at least one of the measures of mis-allocation. In the (very) restricted class of problems with identical marginal costs for different tasks, the modified measure is monotonous and unbiased signals lead indeed to the lowest misallocation. But what if marginal costs differ? To answer this question, we compute the signal which maximises the surplus for a given signal-noise ratio. If the modified measure is monotonous in the larger class, the result must be an unbiased signal. If the signal is biased, the modified measure is not monotonous for different marginal costs.

\section{Mis-allocation minimising signals}

What is the signal which leads to the lowest mis-allocation in a class with a given signal-noise ratio? 
To answer this question, the surplus needs to be maximised while holding the signal-noise ratio constant. Calculating the solution is involved: First, the problem is re-parameterised. Second, the solution to the re-parameterised problem is found to be the eigenvector of a matrix which depends on costs, benefit, risk aversion, and signal-noise ratio. Finally, the solution is transformed back. Details of the proof can be found in the appendix, the solution is summarised in the following theorem.

Theorem 1 (Signal leading to lowest mis-allocation). The signal vector $b^{*}$, which maximises the surplus in the class with a given signal-noise ratio $\rho$, is

$$
b^{*}=k \cdot\left(2 \frac{r}{\rho} C+I\right)^{-1} \beta, \text { with } k:=\sqrt{\frac{\rho \sigma^{2}}{\beta^{\prime}\left(2 \frac{r}{\rho} C+I\right)^{-2} \beta}} .
$$

The role of $k$ is to standardise the length of $b^{*}$ so that the vector indeed belongs to the class with the signal-noise ratio $\rho$.

We have thus identified the signal with the lowest mis-allocation for a given signal-noise ratio. But it is not obvious when this signal is unbiased or biased for the benefit. The answer is given by the following corollary. The proof is based on equating the surplus maximising signal $b^{*}$ from theorem 1 with the benefit vector $\beta$-details can be found in the appendix.

Corollary 1 (Optimality of unbiased signals). An unbiased signal maximises surplus, if and only if effort costs are identical for different tasks: $C=c I$ with $c \in$ $\mathbb{R}$.

This corollary tells us when the intuition works that a congruent signal leads to low mis-allocation: The marginal costs need to be identical. If costs are not identical, the optimal signal coefficient vector generally depends on the costs and the unbiased signal is not optimal anymore. In this case, the intuition fails and none of the introduced measures is monotonous because a signal different from the unbiased signal leads to a higher surplus. A respective example is given in the following section. 


\section{Biased but optimal signals}

This section gives a simple example with two efforts, where the effort costs differ and the optimal signal is biased. Consider the cost matrix

$$
C=\left(\begin{array}{cc}
c_{1} & 0 \\
0 & c_{2}
\end{array}\right) \text { with } c_{1}>c_{2}
$$

Using theorem 1, we can now compute the optimal signal:

$$
b^{*}=k\left(2 \frac{r}{\rho} C+I\right)^{-1} \beta=k\left(2 \frac{r}{\rho}\left(\begin{array}{cc}
c_{1} & 0 \\
0 & c_{2}
\end{array}\right)+I\right)^{-1} \beta=k\left(\begin{array}{cc}
\frac{\rho}{2 r c_{1}+\rho} & 0 \\
0 & \frac{\rho}{2 r c_{2}+\rho}
\end{array}\right) \beta
$$

where $k$ ensures that the signal-noise ratio remains constant. Summarising, the optimal signal vector is:

$$
b^{*}=k \rho\left(\begin{array}{c}
\frac{\beta_{1}}{2 r c_{1}+\rho} \\
\frac{\beta_{2}}{2 r c_{2}+\rho}
\end{array}\right) .
$$

If we divide the first component of this vector by the second component to see the relative importance of the first effort on the signal in comparison to the second effort, the standardisation factor $k$ drops out and we get:

$$
\frac{b_{1}^{*}}{b_{2}^{*}}=\frac{c_{2} 2 r+\rho}{c_{1} 2 r+\rho} \frac{\beta_{1}}{\beta_{2}} .
$$

Thus, the marginal effect of the first effort on the expected signal is not the same as on the benefit. Rather, the optimal signal is biased and honours the cheaper effort $e_{2}$ more than an unbiased signal. What is gained by inducing the agent to exert the cheaper instead of the more expensive effort? The intuition underlying this result is the following. When an unbiased signal is used, the agent allocates effort as if the aim was to provide an overall lower benefit level. The true benefit function, however, has not changed and hence effort re-allocation can increase it. In particular, cheaper effort can be induced in place of more expensive effort. Due to the quadratic cost function giving up a little of expensive effort buys a lot of cheap effort and due to the linear benefit function this effort allocation leads to higher benefit levels. Thus, a signal which puts slightly more weight on the cheaper effort improves the allocation in comparison to an unbiased signal. It is essential for this argument that effort levels are below the first-best levels. So, the presence of uncertainty plays a crucial role for this effect. The larger the signalnoise ratio $\rho$ or the smaller the risk aversion $r$, the closer the optimal signal is to an 
unbiased signal; if the signal becomes very informative $(\rho \rightarrow \infty)$ or the risk aversion decreases $(r \rightarrow 0)$, the optimal signal approaches an unbiased signal. Hence, the disadvantage of using a congruent signal aggravates, the higher the effect of uncertainty. In practice, this implies that the remuneration of workers with little influence on a signal (low signal-noise ratio) should be based on correcting signals to a larger degree than the remuneration of workers with more influence.

The result that the more costly effort needs to be reduced by the signal to obtain the optimal effort allocation is an artifact of the linearity of the benefit function and the quadratic cost function. It is, however, generally true that the desired effort ratio is unlikely to be achieved with an unbiased signal if uncertainty is involved so that effort exertion is below first-best levels.

\section{Conclusion}

In multiple-task models, effort mis-allocation has to be considered when designing an incentive scheme. Despite this commonplace observation, our knowledge about the effects which lead to a distorted allocation of effort between tasks is still sparse. In particular, the effect of signal properties on the allocation are not fully understood, yet. Intuitively, a congruent signal seems to be an ideal candidate for low or even no mis-allocation because the relative marginal effects of effort on the signal are identical to the relative marginal benefits. As we have seen, this intuition fails already in a relatively simple framework: Congruent signals generally don't lead to undistorted effort allocations between different tasks.

Accordingly, mis-allocation measures which assign the lowest mis-allocation to congruent signals are problematic. But this concerns most mis-allocation measures (e.g. Feltham Xie 1994, Datar Kulp Lambert 2001, Baker 2002) and practically leaves us with no sensible approach to directly quantify mis-allocation. ${ }^{3}$ Without mis-allocation measure, the trade-off between mis-allocation and uncertainty, which is thought to underpin the value of signals in multiple task settings, cannot be formalised. Likewise, the value of a signal cannot be determined without computing the surplus which it generates. Overall, it is thus desirable to adapt one of the existing or create a new mis-allocation measure so that the problem of optimal biased signals is accounted for. The properties of mis-allocation measures

\footnotetext{
${ }^{3}$ Indirectly, mis-allocation can be quantified by its consequences on surplus (see Schnedler 2002).
} 
defined in this article (invariance and monotonicity) may provide some guidelines - possibly even for an axiomatic approach.

The finding, that unbiased signals might lead to distortion, does not only put in question approaches how to measure mis-allocation, it also explains the usage of additional performance signals when unbiased signals are available. If for example the objective of a firm is the shareholder value, it might deliberately refrain from using the shareholder value as the only performance signal to motivate a manager and flank it by other accounting information such as sales or revenue. To explain the value of the additional signal, it has been suggested that the signal might reduce uncertainty and hence the wage bill for the risk-averse manager (Datar, Kulp, and Lambert 2001, Baker 2000 and 2002, Schnedler 2002). Alternatively, the design of the incentive scheme might be employed as a commitment device to threaten other firms in an oligopolistic market (Sklivas 1987, Fershtman and Judd 1987). This article brings forward another complementary explanation which solely arises from the incentive problem: The additional signal is used to correct for a mis-allocation of effort.

\section{References}

BAKER, G. (2000): “The Use of Performance Measures in Incentive Contracting," American Economic Review, 90(2), 415-420.

(2002): "Distortion and Risk in Optimal Incentive Contracts," Journal of Human Resources, 37(4), 728-751, Special Issue on Designing Incentives to Promote Human Capital.

Breuer, W. (1993): "Linearität und Optimalität in Ökonomischen AgencyModellen (Eine Anmerkung)," Zeitschrift für Betriebswirtschaft, 63(10), 1073-1076.

DAtAR, S., S. KulP, And R. LAmbert (2001): "Balancing Performance Measures," Journal of Accounting Research, 39(1), 75-92.

Feltham, G. A., And J. XIE (1994): "Performance Measure Congruity and Diversity in Multi-Task Principal/Agent Relations," The Accounting Review, 69(3), 429-453. 
Fershtman, C., AND K. L. JUdD (1987): "Equilibrium Incentives in Oligopoly," The American Economic Review, 77(5), 927-940.

Holmström, B. (1979): "Moral Hazard and Observability," Bell Journal of Economics, 10(1).

HolmströM, B., AND P. Milgrom (1987): "Aggregation and Linearity in the Provision of Intertemporal Incentives," Econometrica, 55(2), 303-328.

(1991): "Multitask Principal-Agent-Analysis: Incentive Contracts, Asset Ownership, and Job Design," Journal of Law, Economics, and Organization, 7, 24-52.

KERR, S. (1975): “On the Folly of Rewarding A While Hoping for B," Academy of Management Journal, 18, 769-783.

KR ÄKEL, M. (1999): Organisation und Management. Mohr Siebeck, Tübingen, Chapter B: Hidden-action im LEN-Modell, pp. 62-77.

Macho-Stadler, I., And D. Perez-Castrillo (1997): An Introduction to the Economics of Information: Incentives and Contracts. Oxford University Press, Oxford.

MirrleES, J. A. (1999): "The Theory of Moral Hazard and Unobservable Behavior (Part 1)," Review of Economic Studies, 66, 3-21, reprint of a manuscript from 1975.

Prendergast, C. (1999): “The Provision of Incentives in Firms," Journal of Economic Literature, XXXII, 7-63.

(2000): "What Trade-Off of Risk and Incentives?," American Economic Review, 90(2), 421-425.

S AlAnIÉ, B. (1998): Economics of contracts. MIT Press, Massachussets.

SCHNEDLER, W. (2002): "The Value of Information in Hidden Action Models," Ph.D. thesis, Bonn University.

SklivaS, S. D. (1987): “The Strategic Choice of Managerial Incentives," The RAND Journal of Economics, 18(3), 452-458. 
Spremann, K. (1987): “Agent and Principal," in Agency Theory Information and Incentives, ed. by G. Bamberg, and K. Spremann, pp. 3-37. Springer, New York, Berlin, London, and Tokyo.

WAGENHOFer, A., AND R. EWERT (1993): "Linearität und Optimalität in Ökonomischen Agency Modellen," Zeitschrift für Betriebswirtschaft, 63, 373391. 


\section{A Surplus maximising signal}

Maximise the surplus in $b$ while holding the signal-noise ratio constant:

$$
\max _{b} \phi\left(b, \sigma^{2}\right) \text { such that } b^{\prime} b=\rho \sigma^{2} \Leftrightarrow \max _{\left\{b \mid b^{\prime} b=\rho \sigma^{2}\right\}} \frac{1}{4} \frac{b^{\prime} C^{-1} \beta \beta^{\prime} C^{-1} b}{b^{\prime}\left(C^{-1}+\frac{2 r}{\rho} I\right) b},
$$

where the last expression was obtained by replacing the variance $\sigma^{2}$. Note, that the solution to the problem does not depend on the length of $b$ : If $b$ solves the problem so does $k b$. We can thus drop the restriction on the length of $b$ for the moment, find any solution to the problem and standardise the solution to the correct length later.

Define $\tilde{C}:=C\left(\frac{2 r}{\rho} C+I\right)^{-1}$, then the inverse exists and is equal to the matrix in the numerator: $\tilde{C}^{-1}=\left(C^{-1}+\frac{2 r}{\rho} I\right)$. Because $\tilde{C}$ is symmetric and positive definite, we can decompose it ( $\tilde{C}=P \Lambda P^{\prime}$ where $\Lambda$ is a diagonal matrix and $P$ and $P^{\prime}$ are projector matrices) and define $\tilde{C}^{-\frac{1}{2}}:=P \Lambda^{-\frac{1}{2}} P$, where the inverse ratio and squareroot is taken of all diagonal elements in $\Lambda$. Define $\tilde{b}:=\tilde{C}^{-\frac{1}{2}} b$ and consider the transformed problem:

$$
\max _{\tilde{b}} \frac{\tilde{b}^{\prime} \tilde{C}^{\frac{1}{2}} C^{-1} \beta \beta^{\prime} C^{-1} \tilde{C}^{\frac{1}{2}} \tilde{b}}{\tilde{b}^{\prime} \tilde{b}} .
$$

Fix the length of $\tilde{b}$ to one: $\tilde{b}^{\prime} \tilde{b}=1$. Then, the respective Lagrangian is:

$$
L(\tilde{b}, k)=\tilde{b}^{\prime} \tilde{C}^{\frac{1}{2}} C^{-1} \beta \beta^{\prime} C^{-1} \tilde{C}^{\frac{1}{2}} \tilde{b}-\lambda\left(\tilde{b}^{\prime} \tilde{b}-1\right) .
$$

The corresponding first-order conditions are:

$$
\left(\left(\tilde{C}^{\frac{1}{2}}\right)^{\prime} C^{-1} \beta \beta^{\prime} C^{-1} \tilde{C}^{\frac{1}{2}}-\lambda I\right) \tilde{b}=0 \text { and } \tilde{b}^{\prime} \tilde{b}=1
$$

The first condition is an eigenvalue problem; to obtain $\tilde{b}$, we have to find the eigenvalues $\lambda$ of the matrix $\tilde{C}^{\frac{1}{2}} C^{-1} \beta \beta^{\prime} C^{-1} \tilde{C}^{\frac{1}{2}}$. By defining $x:=\left(\tilde{C}^{\frac{1}{2}}\right)^{\prime} C^{-1} \beta$, the matrix can be re-written as $x x^{\prime}$ and it becomes apparent that the matrix is symmetric and of rank one. Due to the latter, there can only be one non-zero eigenvalue. This value can be obtained using the trace-operator $(\operatorname{tr}(\cdot))$ :

$$
\mathrm{EV}\left(x x^{\prime}\right)=\operatorname{tr}\left(x x^{\prime}\right)=\operatorname{tr}\left(x^{\prime} x\right)=x^{\prime} x
$$


Replacing this eigenvalue in the eigenvalue problem, we get:

$$
\left(x x^{\prime}-x^{\prime} x I\right) \tilde{b}=0 .
$$

Obviously, $\tilde{b}=k x, k \in \mathbb{R}$ is a solution to this problem. Again due to the rank of $x x^{\prime}$, it is also the only solution. To recover the solution in the original problem, we have to reverse the transformation:

$$
b^{*}=\tilde{C}^{\frac{1}{2}} \tilde{b}=k \tilde{C}^{\frac{1}{2}} x=k \tilde{C}^{\frac{1}{2}} \tilde{C}^{\frac{1}{2}} C^{-1} \beta=k \tilde{C}^{\prime} C^{-1} \beta=k(2 \underset{\rho}{\stackrel{r}{\rho}} C+I)^{-1} \beta .
$$

Finally, we are free to choose the length of the vector as $k:=\sqrt{\frac{\rho \sigma^{2}}{\beta^{\prime}\left(2 \frac{r}{\bar{\rho}} C+I\right)^{-2} \beta}}$. Then, $\left(b^{*}\right)^{\prime} b^{*}=\rho \sigma^{2}$ and the signal is indeed a member of the signal-noise ratio class $\rho$.

\section{B Optimality of unbiased signals}

By theorem 1, it holds that an unbiased signal maximises surplus (given a constant signal-noise ratio) if and only if

$$
\left(2 \frac{r}{\rho} C+I\right)^{-1}=k I, k \in \mathbb{R} .
$$

This equality can be rewritten as $2 \frac{r}{\rho} C+I=\frac{1}{k} I$. Hence, an unbiased signal maximises surplus only if $C=c I$. If on the other hand $C=c I$ then the constant $k$ can be chosen so that the equality (12) holds $\left(k=2 \frac{r c}{\rho}+1\right)$ and hence identical costs imply that unbiased signals maximise surplus. 